\title{
Studies on the properties of zirconia films prepared by direct current reactive magnetron sputtering
}

\author{
M. H. Suhail, G. Mohan Rao, andS. Mohan \\ Instrumentation and Services Unit, Indian Institute of Science, Bangalore 560012, India
}

\begin{abstract}
This paper deals with the preparation of zirconia films by direct current reactive magnetron sputtering. The films were annealed in ambient atmosphere at temperatures ranging from 300 to 850 " $C$. The as deposited films were found to be amorphous and a crystalline structure developed at 400 " $\boldsymbol{C}$. The refractive index and extinction coefficient were found to be 1.93 and $0.5 \times 10^{-3}$ at $600 \mathrm{~nm}$ for the as deposited films and they changed to 1.71 and $2.5 \times 10^{-3}$ on annealing at 850 "C. The optical constants were found to be constant in the wavelength region beyond $400 \mathrm{~nm}$, below which dispersion was observed.
\end{abstract}

\section{INTRODUCTION}

Zirconia $\left(\mathrm{ZrO}_{2}\right)$ films are useful in diverse applications such as optical coatings, thermal barrier layers' etc. This is due to the high-refractive index, high melting point, hardness and corrosion resistance of these films. An important property of zirconia which affects its applications is polymorphism. At room temperature, it is monoclinic. On heating to 1100 " $\mathrm{C}$ it transforms to tetragonal and above 2285 "C it becomes cubic. ${ }^{2}$ By appropriately mixing a sufficient quantity of materials such as $\mathrm{Y}_{2} \mathrm{O}, \mathrm{CaO}$, and $\mathrm{Al}, \mathrm{O},{ }^{3-5}$ the high temperature phase can be made to exist even at room temperature.

Zirconia films have been deposited by various techniques, ${ }^{2,6-9}$ reactive sputtering being one of the most widely used. Reactive magnetron sputtering offers good control over the deposition rate and film composition, and minimizes target poisoning. However, this technique has not yet been well studied for this purpose. The aim of this study was to prepare zirconia films by direct current (dc) reactive magnetron sputtering and study the effect of deposition parameters on the structure and properties of these films. The films were annealed at different temperatures and the effect of annealing on the optical properties and structure has been investigated.

\section{EXPERIMENTAL}

Zirconia films were deposited by dc reactive magnetron sputtering from a pure zirconium (99.8\%) disk of $152 \mathrm{~mm}$ diam (Good fellow metals). The zirconium disk was mechanically clamped to a water cooled copper cathode and the assembly was used as sputtering target. The magnetron cathode was homemade, similar to the one used by Mohan Rao and Mohan." A variable power supply ( $1 \mathrm{kV}$ and $6 \mathrm{~A}$ ) was used in controlling the glow discharge. The pumping system was a diffusion pumprotary pump combination with a liquid nitrogen trap, capable of giving an ultimate vacuum of $10^{-6}$ Torr. IOLAR 2 grade oxygen $(99.99 \%)$ and argon $(99.999 \%)$ have been used as reactive and sputtering gases, respectively. The pressure was monitored with a PiraniPenning gauge combination calibrated against argon gas us- ing a Mcleod gauge. Presputtering was done for $\mathbf{3 0} \mathrm{min}$. in pure argon to remove the oxide layer that had formed on the target surface during exposure to air. After presputtering, the reactive gas was introduced through a needle valve and was stabilized at a desired pressure. Quartz substrates (25 $\mathrm{mm}$ diam), after cleaning with Balzer's substrate cleaner, were loaded into the chamber. Annealing of deposited films was performed in air in the temperature range $300-850^{\circ} \mathrm{C}$. The spectral characteristics of the films were measured with a Hitachi (model 330) spectrophotometer. The refractive index $(n)$, extinction coefficient $(k)$ and thickness of the films have been calculated using an envelope technique due to Swanepoel. ${ }^{11}$ An x-ray diffractometer technique using Co $K \alpha$ radiation was used to study the structure of these films.

\section{RESULTSAND DISCUSSION}

Figure 1 shows the variation of deposition rate as the oxygen partial pressure is varied in the system. The deposition decreases slowly up to a pressure of $3 \times 10^{-5}$ Torr, then abruptly drops and beyond this pressure almost remains constant. This abrupt drop in deposition rate is attributed to target poisoning due to oxide formation, as the corresponding oxide has lower deposition rate. ${ }^{12}$ A similar effect has been observed in the radio frequency (rf) sputtering of $\mathrm{TiO}_{2}$ films. ${ }^{13}$ Since stoichiometric oxides are formed when the process is carried out at the critical oxygen pressure where the target oxidation just starts taking place, ${ }^{14}$ all the films were deposited at an oxygen pressure of $3 \times 10^{-5}$ Torr and a total pressure of $2 \times 10^{-2}$ Torr.

$\mathrm{X}$-ray diffraction patterns of the as-deposited and annealed films are shown in Fig. 2. The as-deposited films are amorphous and crystallize during annealing. On annealing at 400 " $\boldsymbol{C}$ for $1 \mathrm{~h}$, the film becomes crystalline with the monoclinic phase as the major component with peaks $M(11 \overline{1})$, $M(111), M(200), M(221)$, and $M(131)$, with just one peak corresponding to tetragonal phase, $\mathrm{T}$ (111). After annealing at 700 " $\boldsymbol{C}$, the monoclinic phase dominates [see the $\mathbf{M}(200)$ line] with the intensity of the tetragonal phase (111) reflection relatively reducing. At $850^{\circ} \mathrm{C}$ the tetragonal phase is further reduced. This change in the phases at different an- 


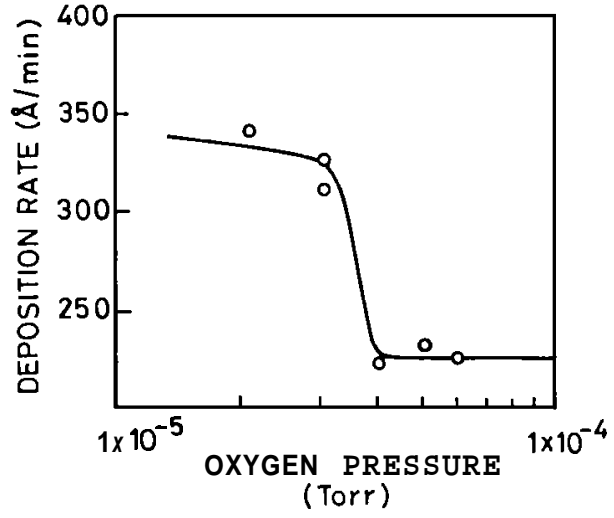

Fig. 1. Variation in deposition rate as a function of oxygen pressure. (sputtering pressure $2 \times 10^{-2}$ Torr).

nealing temperatures is similar to that observed by Debsikdar ${ }^{15}$ in zirconia films deposited by chemical methods. Krishna et al. ${ }^{16}$ also studied the effect of annealing in the temperature range $500-850^{\circ} \mathrm{C}$ on evaporated $\mathrm{ZrO}_{2}$ films. They could see the cubic phase also at $500^{\circ} \mathrm{C}$, which has not been noticed in the present work. Otherwise the existence of tetragonal and monoclinic phases at higher temperatures observed in the present work is similar to that noticed by Krishna et al. ${ }^{16}$ The absence of cubic phase in the sputtered films reported here might be because of the difference in the technique of deposition. This explanation on the basis of process dependence is supported by Martin et al. ${ }^{9}$ who observed the monoclinic phase at a temperature as low as $\mathbf{3 0 0}{ }^{\circ} \mathrm{C}$ in $\mathrm{ZrO}_{2}$ films prepared by ion assisted deposition and Rujkorakarn and Sites, ${ }^{17}$ who obtained monoclinic and tetragonal phases at $450{ }^{\circ} \mathrm{C}$ and monoclinic and cubic at 975 " $\mathrm{C}$ in ion beam sputtered $\mathrm{ZrO}_{2}$ films. Since the reported data on such crystalline transitions for zirconia films is scattered and some times contradictary, it may be concluded that the phenomena is highly process, deposition parameter and thickness dependent.

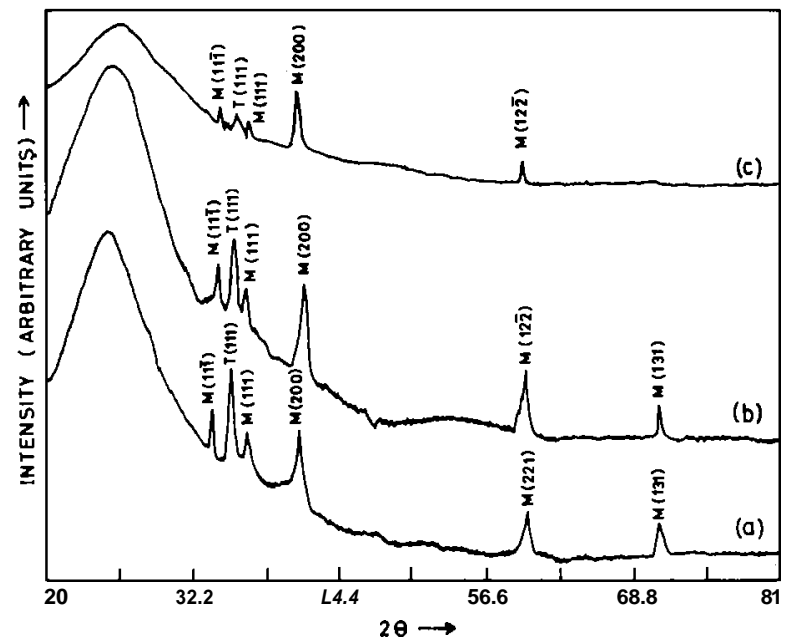

FIG. 2. X-ray diffraction patterns for zirconia films annealed at (a) $400^{\circ} \mathrm{C}$, (b) $700^{\circ} \mathrm{C}$, (c) $850^{\circ} \mathrm{C}$

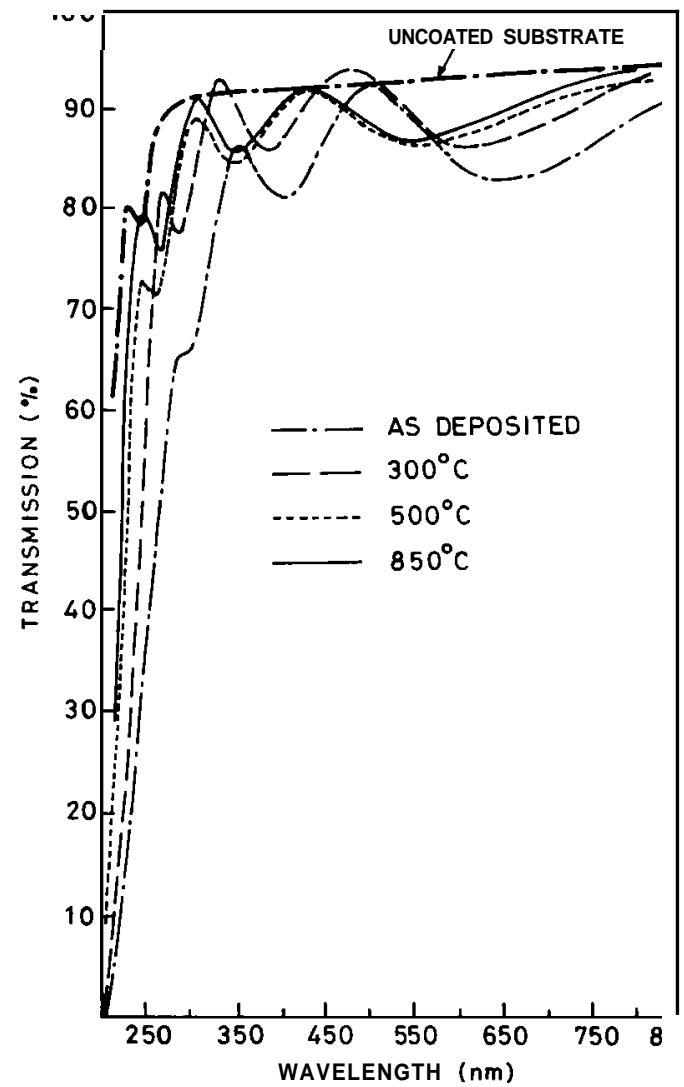

FIG. 3. Transmission spectra of as deposited and annealed zirconia films.

The optical transmission of the as deposited and annealed films is shown in Fig. 3. It is seen that as the annealing temperature is increased, the interference pattern shifts to lower wavelength indicating increase in oxidation, changes in packing density and microstructure of the films. The reduction in the refractive index of the films is evident from the position of transmission maxima and minima.

The refractive index $(n)$ and extinction coefficient $(k)$ of the films as a function of wavelength for different annealing temperatures are shown in Fig. 4. The as-deposited films show a high refractive index which decreases with increase in annealing temperature. The $n$ value decreases from $\mathbf{1 . 9 3}$ (at $600 \mathrm{~nm}$ ) for the as deposited film to $\mathbf{1 . 7 1}$ for the film annealed at $850^{\circ} \mathrm{C}$. A similar variation has been observed in films prepared by reactive electron beam evaporation. ${ }^{16}$ The refractive index changed from $\mathbf{1 . 9}$ for as-deposited film to 1.71 for the film annealed at 850 "C. This has been explained on the basis of structural transitions and changes in packing density. The refractive index of the films reported here is slightly lower than the bulk value (2.2) and compares favourably with the values reported for zirconia films produced by other techniques. ${ }^{6.9 .17-19}$ Klinger and Carniglia ${ }^{6}$ by using electron beam evaporation obtained films with refractive index in the range 1.95-2.25. Martin et al. ${ }^{9}$ reported a refractive index in the range 1.72-2.0 in electron beam evaporated $\mathrm{ZrO}_{2}$ films and $\mathbf{2 . 2 4}$ in ion assisted deposited films. The ion beam sputtered films prepared by Rujkorakarn and Sites $^{17}$ have a refractive index of 2.1 at $(800 \mathrm{~nm})$ in 

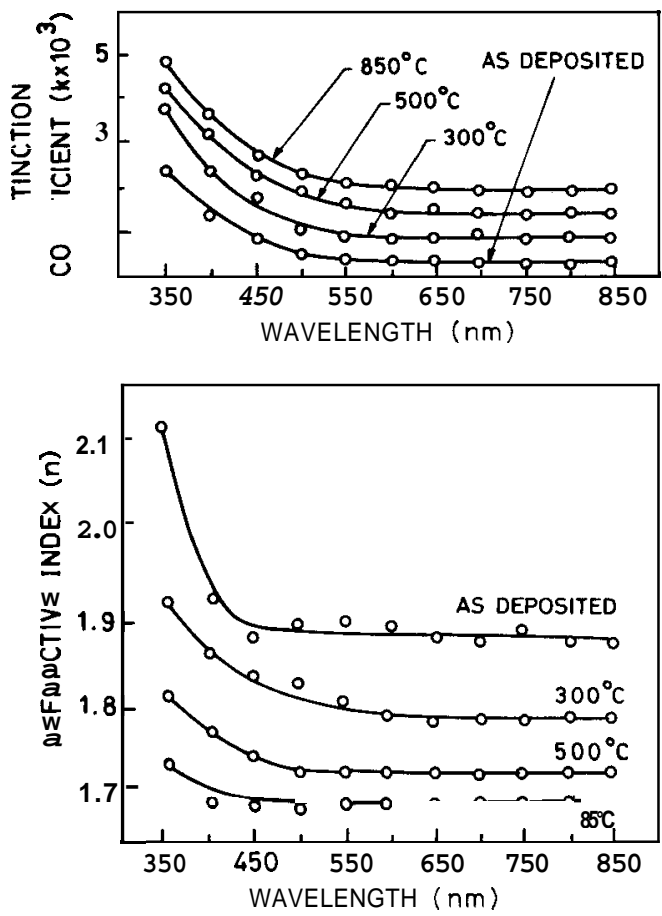

FIG. 4. Variation of refractive index and extinction coefficient as a function of wavelength for as deposited and annealed zirconia films.

the as-deposited condition and decreased to 1.98 after annealing to 850 "C. Pawlewicz et al. ${ }^{\text {s }}$ by, using rf diode reactive sputtering, obtained films with a refractive index of 2.14-2.47 in the wavelength range $(250-1060 \mathrm{~nm})$. Apparao et al. ${ }^{19}$ deposited $\mathrm{ZrO}_{2}$ films by electron beam evaporation having a refractive index greater than 2 , but they correspond to a lower wavelength region $(240-400 \mathrm{~nm}$ ). Another feature of the refractive index is that in the wavelength range above $450 \mathrm{~nm}$, it is nearly constant, but shows dispersion at lower wavelengths. However, this dispersion continuesto be present even after annealing, though the variation is not as high as observed in the as deposited films. This may be due to phase transition exhibited by the films at different temperatures as observed by Rujkorakarn and Sites ${ }^{17}$ for ion beam sputter deposited zirconia coatings. Figure 4 also shows the effect of annealing on the extinction coefficient $(k)$. The $(k)$ of the as deposited film at $600 \mathrm{~nm}$, was $0.5 \times 10^{-3}$ which increased on annealing at $850^{\circ} \mathrm{C}$ to $2.5 \times 10^{-3}$. Similar observationshave been made by Rujkorakarn and Sites" who observed increase in $(\boldsymbol{k})$ from
$1.5 \times 10^{-3}$ for as deposited films ( $800 \mathrm{~nm}$ ) to $4 \times 10^{-3}$ for films annealed at $850^{\circ} \mathrm{C}$ which has been attributed to the phase transition. ${ }^{17}$

\section{CONCLUSIONS}

In this study good optical quality zirconia films have been deposited by the reactive dc magnetron sputtering technique. The variation in deposition rate has been shown to be dependent on the oxygen partial pressure. The as deposited films showed a refractive index of $2.13(350 \mathrm{~nm})$ and extinction coefficient of $5 \times 10^{-3}$. The as deposited films are amorphous and the transition to crystalline phase was observed at around $400{ }^{\circ} \mathrm{C}$. The phase transition was found to be strongly dependent on deposition technique and process parameters. The optical constants were found to be constant in the visible and infrared regions and showed dispersion in ultraviolet. This dispersion was found to decreaseafter annealing.

\section{ACKNOWLEDGMENTS}

The authors thank M. G. Krishna and other colleagues in the thin films laboratory for valuable discussions.

${ }^{1}$ K. C. Fancey and A. Mathews, J. Vac. Sci. Technol. A 4,2656 (1986).

${ }^{2}$ F. Jones, J. Vac. Sci. Technol. A 6, 3088 (1988).

${ }^{3}$ F. Lange, J. Mater. Sci. 17, 240 ( 1982).

${ }^{4}$ F. Lange, J. Mater. Sci. 17, 247 (1982).

${ }^{5}$ P. Duwez and F. Odell, J. Am. Ceram. Soc. 33, 274 (1950).

${ }^{6}$ V. V. Klechkovskaya, V. I. Khitrova, S. I. Sagitov, and S. A. Semiletov, Sov. Phys. Crystallogr. 25, 636 (1980).

${ }^{7}$ R. E. Klinger and C. K. Carniglia, Appl. Opt. 24,3185 (1985).

${ }^{8}$ E. N. Farabaugh, A. Feldman, J. Sun, and Y. N. Sun, J. Vac. Sci. Technol. A 5, 1671 (1987).

${ }^{9}$ P. J. Martin, R. P. Netterfield, and W. G. Sainty, J. Appl. Phys. 55, 235 (1984).

${ }^{10}$ G. Mohan Rao and S. Mohan, Vacuum 40,313 (1990).

${ }^{11}$ R. Swanepoel, J. Phys. E 16, 1214 (1983).

${ }^{12}$ M. Cantagrel and M. Marchal, J. Mater. Sci. 8, 1711 (1973).

${ }^{13}$ F. Donaghey and I. G. Geraghty, Thin Solid Films 38,271 (1976).

${ }^{14}$ S. Schiller, U. Heisig, and K. Goedicke, Thin Solid Films 118, 255 (1984).

${ }^{15}$ J. C. Debsikdar, J. Noncryst. Solids 87, 343 (1986).

${ }^{16}$ M. Ghanashaym Krishna, K. Narasimharao, and S. Mohan, Appl. Phys. Lett. 57, 557 (1990).

${ }^{17}$ R. Rujkorakarn and J. R. Sites, J. Vac. Sci. Technol. A 4, 568 (1986).

${ }^{18}$ W. T. Pawlewicz, D. D. Hays, and P. M. Martin, Thin Soild Films 73, 169 (1980).

${ }^{19}$ K. V. S. R. Apparao, N. K. Sahoo, and T. C. Bagchi, Thin Solid Films 129, L71 (1985). 